\title{
The effects of takeaway (fast) food consumption on UK adolescent's diet quality
}

\author{
A. Taher and C.E.L. Evans \\ Nutritional Epidemiology Group, School of Food Science and Nutrition, University of Leeds, Leeds, LS2 9JT, UK
}

Overconsumption of energy dense foods derived from fast and convenience food outlets is stated to be a risk factor for obesity and diabetes among young people ${ }^{(1)}$. Consumption of takeaway meals and purchase of food from outside the home rather than preparation of food at home is found to be negatively associated with diet quality ${ }^{(2)}$. The aim of this project is to evaluate the association between the frequency of consuming takeaway meals and meals out and diet quality of UK adolescents aged 11-18 year old. Previous research has assessed individual macro and/or micro nutrients, however the need for higher quality data to strengthen the evidence is required. Therefore, the diet quality index for adolescents (DQI-A) tool has been used to assess diet quality where adolescent's food intake was based on 4-day dairy records obtained from the NDNS rolling programme years 1-6. The DQI-A relies on three main components, particularly quality, diversity and equilibrium which reflect the degree of adherence of an adolescent diet with Food Based Dietary Guidelines (FBDG) ${ }^{(3,4)}$. The high consumption of takeaway meals at home and consuming meals out $(1-2$ times/ week or more) were reported by $28.8 \%(\mathrm{n}=589)$ and $24.3 \%(\mathrm{n}=496)$ of both male and female adolescents aged $11-18$ years respectively. In terms of the diet quality index score, UK adolescents have a poor diet quality score of $25.7 \%$ out of $100 \%$ with significant differences observed between high and low takeaway consumers. In addition, significant differences were observed between high and low takeaway consumers among all other DQI-A components and sub-components $(\mathrm{p}<0.05)$, except for the diet adequacy subcomponent $(\mathrm{DAx})$. Nevertheless, high takeaway consumers were found to have a higher food energy intake by $51 \cdot 3 \mathrm{kcal}(\mathrm{CI}=$ $3.79,98.73, \mathrm{p}=0.03$ ) before and after adjusting for age, gender and equivalised household income. The results for high consumption of meals-out were attenuated and not statistically significant for individual components.

\begin{tabular}{|c|c|c|c|c|c|c|c|c|c|c|}
\hline \multirow{3}{*}{ Diet quality components, age $\&$ energy } & \multirow{2}{*}{\multicolumn{2}{|c|}{$\frac{\text { Total sample }}{\mathrm{n}=2045}$}} & \multirow{2}{*}{\multicolumn{2}{|c|}{$\begin{array}{l}\text { High takeaway } \\
\text { consumers } \\
\mathrm{n}=589\end{array}$}} & \multirow{2}{*}{\multicolumn{2}{|c|}{$\begin{array}{l}\text { Low takeaway } \\
\text { consumers } \\
\mathrm{n}=1456\end{array}$}} & \multicolumn{2}{|c|}{$\underline{\text { Unadjusted analysis }}$} & \multicolumn{2}{|c|}{$\underline{\text { Adjusted analysis }}$} \\
\hline & & & & & & & \multirow[b]{2}{*}{$(95 \% \mathrm{CI})$} & \multirow[b]{2}{*}{$\mathrm{P}$-value } & \multirow[b]{2}{*}{$(95 \% \mathrm{CI})$} & \multirow[b]{2}{*}{ P-value } \\
\hline & Mean & $\mathrm{SD}$ & Mean & SD & Mean & SD & & & & \\
\hline DQI-A \% & $25 \cdot 7$ & $14 \cdot 4$ & $23 \cdot 0$ & $14 \cdot 5$ & $26 \cdot 8$ & $14 \cdot 2$ & $-5 \cdot 19,-2 \cdot 45$ & $<0 \cdot 01$ & $-5 \cdot 03,-2 \cdot 08$ & $<0.01$ \\
\hline DQc $\%$ & -9.7 & $27 \cdot 7$ & $-15 \cdot 3$ & $28 \cdot 2$ & $-7 \cdot 4$ & $27 \cdot 2$ & $-10 \cdot 49,-5 \cdot 22$ & $<0 \cdot 01$ & $-9.92,-4.28$ & $<0.01$ \\
\hline DDc \% & $53 \cdot 6$ & $16 \cdot 8$ & $52 \cdot 2$ & $16 \cdot 5$ & $54 \cdot 2$ & $16 \cdot 9$ & $-3 \cdot 66,-0.44$ & $0 \cdot 01$ & $-3.65,-0.26$ & $0 \cdot 02$ \\
\hline DEc \% & $33 \cdot 3$ & $10 \cdot 2$ & $32 \cdot 1$ & $10 \cdot 0$ & 33.7 & $10 \cdot 3$ & $-2 \cdot 54,-0 \cdot 58$ & $<0 \cdot 01$ & $-2 \cdot 50,-0 \cdot 40$ & $<0.01$ \\
\hline DAx $\%$ & $63 \cdot 2$ & 11.8 & $63 \cdot 0$ & $11 \cdot 7$ & $63 \cdot 3$ & 11.9 & $-1.43, \quad 0.83$ & 0.61 & $-1.22, \quad 1.15$ & $0 \cdot 96$ \\
\hline DEx \% & 18.5 & $9 \cdot 4$ & $19 \cdot 4$ & $9 \cdot 6$ & $18 \cdot 1$ & $9 \cdot 3$ & $0.42, \quad 2 \cdot 21$ & $<0 \cdot 01$ & $0.49, \quad 2.36$ & $<0.01$ \\
\hline Age (year) & $14 \cdot 6$ & $2 \cdot 2$ & $14 \cdot 6$ & $2 \cdot 2$ & $14 \cdot 5$ & $2 \cdot 2$ & $-0.15, \quad 0.27$ & 0.57 & $-0 \cdot 12, \quad 0.33$ & $0 \cdot 38$ \\
\hline Energy (Kcal) & 1758 & 501 & 1809 & 521 & 1738 & 491 & $23 \cdot 27, \quad 119$ & $<0 \cdot 01$ & $3.79, \quad 98.73$ & 0.03 \\
\hline
\end{tabular}

DQI-A, Diet Quality Index for Adolescents; DQc, Diet quality component; DDc, Diet diversity component, DEc, Diet equilibrium component; DAx, Diet adequacy sub-component; DEx, Diet excess sub-component

Adolescents who consumed high takeaway meals and meals out had a lower overall DQI-A score by 3.8 and 1.9 percent $(\mathrm{CI}=-$ $5 \cdot 19,-2.45$ and $-3 \cdot 37,-0.46, \mathrm{p}<0.01$,$) respectively. Similarly, after adjusting for age, gender and equivalised household income,$ DQI-A score was observed to be lower for both high takeaway and meal out consumers by $3 \cdot 5$ and $2 \cdot 5$ percent $(\mathrm{CI}=-5 \cdot 03$, $-2 \cdot 08$ and $-4.08,-0.90$, p-values < 0.01, ) respectively (data not shown in table for high meal out consumers). In conclusion, these results confirm that high consumption of takeaway meals and meals-out have a negative impact on diet quality of UK adolescents.

1. Poti JM, Duffey KJ \& Duffey KJ (2014) Am J Clin Nutr 99, 162-71.

2. Woodruff SJ \& Hanning RM (2009) Diet Pract Res J 70, 118-24.

3. Vyncke K, Fernandez EC, Fajo-Pascual M et al. (2013) Brit J Nutr 109, 206-78.

4. Huybrechts I, Vereecken C, De Bacquer D et al. (2014) Brit J Nutr 104, 135-44. 